\title{
Cancer pain as a meaningful aspect of the oncological treatment
}

\author{
Izabela Marzec', Kinga Grabska', Marta M. Fudalej, ${ }^{2,3}$, \\ Anna M. Badowska-Kozakiewicz ${ }^{2}$
}

'Students' Scientific Organization of Cancer Cell Biology, Department of Cancer Prevention, Medical University of Warsaw

${ }^{2}$ Department of Cancer Prevention, Medical University of Warsaw

${ }^{3}$ Doctoral School, Medical University of Warsaw

Head of the Department: Prof. Andrzej Deptała, MD, PhD

Correspondence:

Marta Fudalej Department of Cancer Prevention 02-091 Warsaw, Żwirki i Wigury 81 e-mail:mmfudalej@gmail.com

Received:

17.12.2020

Accepted:

8.01.2021

DOI: 10.24292/01.OR.420080121 Copyright $\odot$ Medical Education. All rights reserved.

\section{ABSTRACT}

Introduction and objective: Pain is the most common and universal symptom among all patients with the oncological disease. Pain significantly reduces the quality of life, hinders decent functioning, and plays a significant role in the deterioration of the mental health of the patient and his close relatives. The study aimed to discuss the mainstreaming of effective pain treatment and to review various assessments and scales (including Brief Pain Inventory, Illness Perception Questionnaire and Numeric Rating Scale) concerning the psychological aspect of pain in selected neoplastic diseases.

State of knowledge: We can distinguish various treatments for pain that can be divided into pharmacological and non-pharmacological methods. Latest studies revealed that pain treatment appears to be more and more meaningful. Various factors might influence pain perception and response to the applied treatment. Among all malignancies, special attention is paid to the pain issue in following cancers: colon cancer, gastric cancer, pancreatic cancer, ovarian cancer, breast cancer and lung cancer, that were described in this paper.

Conclusions: Effective pain relief presents a positive effect, both on the physical and mental state of the patient. It also helps to maintain calm mental health among relatives. Nowadays, integration of the best methods for pain relief that are characterized as humanitarian, easily accessible and effective, seems to be one of the biggest challenges for both oncological and palliative health workers.

Key words: pain, scales, cancer, analgesic treatment, palliative care 


\section{INTRODUCTION}

Pain is one of the most frightening symptoms among patients with the oncological disease. It plays a huge role in their lives, influences their psychosocial health, and significantly reduces the quality of their everyday life [1, 2]. Pain is the most common and universal symptom among all patients with various malignancies. Over $1 / 3$ of patients claim that the cancer-related pain makes it difficult to function properly or is an unbearable aspect of their disease [3]. Pain, by reducing the ability to concentrate or even think effectively, creates difficulties in previously usual activities. The burden of cancer disease and the accompanying pain affects not only the patients themselves, but also presents a large impact on the life and mental health of the patient's closest family members. Assuagement of the pain is a meaningful aspect of oncological therapy. Therefore, in 1986 the World Health Organization (WHO) introduced the analgesic ladder, which is accepted and used all over the world (graph 1) [4]. Integrating the finest ways to manage the pain in a humane, accessible, and effective manner is currently one of the greatest challenges to be dealt with by oncologists and palliative care specialists around the world [5].

The study aimed to discuss the mainstreaming of effective pain treatment and to review various assessments and scales. Articles published since 1987 up to 2020 referring to questionnaires and scales assessing the psychological aspect of the pain in the adult population suffering from cancer found in the PubMed and Elsevier databases were analyzed. Authors focused on breast, ovarian, lung, colorectal, pancreatic, and gastric neoplasms.

\section{PAIN - PATHOPHYSIOLOGY}

Cancer-related pain should be considered a complex phenomenon associated with the interaction between the cancer cells and different systems, including nervous and immune ones [6]. The causes of pain in oncological patients might be divided into cancer- and treatment-induced [7]. Cancer pain is characterized by the mixed pathophysiology encompassing neuropathic and nociceptive components [6,7].

Neuropathic pain is defined as nerve-related, due to its association with the direct injury of the nervous system. In oncological conditions nerve injury occurs commonly after both direct tumor compression or infiltration and treatments, including surgery, chemotherapy, or radiotherapy [8]. Injured pain fibers become hypersensitive and even a minor, innocuous stimulus triggers intensify the pain. This condition, defined as allodynia, along with hyperalgesia, is a fundamental and intractable symptom of neuropathic pain $[9,10]$. Treatment algorithms for cancer-related neuropathic pain are mostly based on noncancer-related neuropathic pain guidelines [11]. Although a few clinical studies support opioids monotherapy, the vast majority recommend analgesic combinations of antidepressants, anticonvulsants, and opioids. An adequate combination may result in a better overall pain outcome and fewer side effects in oncological patients $[11,12]$. Aside from direct nerve injury, neuropathic pain might be induced by chemotherapy usage. Among the most common chemotherapeutic agents causing neuropathic pain, we can distinguish platinum agents, taxanes, and the vinca alkaloids [13]. Because of the absence of effective, widely used treatment options for chemotherapy-induced neuropathic pain, oncological patients might suffer from this condition for several months or years and may never be completely cured [14]. Neither nonsteroidal anti-inflammatory drugs nor opioids produce adequate analgesia levels [15]. Mechanisms responsible for chemotherapy-induced pain include mitochondrial damage, disruption of axonal transport, the release of pro-inflammatory factors, and increased ion channel activity [8]. Extended knowledge about possible mechanisms opens new therapeutic options. For oxaliplatin- and paclitaxel-induced neuropathic pain one of the most promising drugs is duloxetine, which balances neurotransmitter levels in the central nervous system and reduces neuronal hyperexcitability. In the randomized, clinical study duloxetine usage for 5 weeks resulted in a greater pain reduction among patients with chemotherapy-induced painful peripheral neuropathy [16]. Other therapeutic options that are under investigation encompass phosphodiesterase 4 inhibitors. One of them - rolipram alleviates the mechanical paclitaxel-induced allodynia in animal studies. Rolipram mechanism of action is based on the modulation of the cyclic AMP (CAMP) levels involved in the neuropathic pain expansion [17]. In the terms of pro-inflammatory mechanism of neuropathic pain, inhibition of IL-1 and IL- 6 signaling pathways with the usage of anakinra (IL-1 antagonist) or tocilizumab (IL-6 blocker) is proposed $[13,18,19]$.

Nociceptive pain originates from the activation of high-threshold afferents defined as nociceptors. This type of pain is divided into somatic (well localized, associated with nociceptors located in superficial structures) and visceral (worse localized, associated with nociceptors located in organs) [20]. The nociceptive pain is attributable to noxious thermal, mechanical, and chemical stimuli or actual damage to non-neural tissue [21]. In oncological patients, nociception involves dynamic interaction between cancer and the primary afferent nociceptors [14]. Most of the cancer pain arises from the tumor microenvironment encompassing cytokines, tumor necrosis factors, neuroimmune mediators, 
acidosis, and proteolytic activity. Released neuroimmune mediators interact with receptors on peripheral nociceptive nerve terminals $[14,22]$. Additionally, the term inflammatory pain was excluded to describe pain occurring after the sensitization of the nociceptive system by active peripheral inflammation [14]. Nociceptive pain, in contrast to the neuropathic one, is considered to respond well to the opioid treatment [23]. However, multifactorial characteristics of cancer pain and mixed pathophysiology, including nociceptive and neuropathic components require a multimodal treatment approach [6].

\section{PAIN - PREVALENCE AND TREATMENT}

A study published in 2007 provided a systematic review of the incidence of cancer pain during the 40-year follow-up. This study concluded that there was no progress in the treatment of cancer pain during that period [24]. More than 10 years have passed since the publication of that review and recent studies still show that the importance of treating pain in cancer patients only in creases. This assumption was posed based on reports of the improvement of pharmacological pain control and the increase in the global consumption of opioids $[25,26]$.

While assessing pain, many factors should be considered - a precise description of the pain, its type and location, type and severity of the underlying disease, and comorbidities, which may affect the severity of the pain [5]. We cannot forget about the psychological aspect of the pain, strategies of coping with it, how it affects proper functioning in society, and whether there are factors that can relieve the pain [1, 27-29].

The main method of treating cancer pain is a pharmacological one, which is based on opioids. Non-steroidal anti-inflammatory drugs may also be added to the above treatment. Adjuvant analgesics such as glucocorticosteroids, antidepressants, and anticonvulsants are also widely used [4]. Opioids cause their analgesic effect by binding to the opioid receptors - mainly the $\mu$-opioid one in the central nervous system. However, these receptors are also found outside the central nervous system, in the intestinal neurons. Their localization also determines the side effects of opioids like respiratory depression, addiction, constipation, nausea, and vomiting. This should not escape a physician's notice [30].

Non-steroidal anti-inflammatory drugs (NSAIDs) present analgesic, anti-inflammatory and antipyretic effects [31]. They act by inhibiting the activity of cyclooxygenase (COX) and, as a result inhibit the production of prostaglandins, which are known pain mediators, that modulate multiple points along the nociceptive pathway [32].

Effective pharmacological treatment depends on the appropriate selection of drugs, as well as proper administration and adaption of doses to the patient's needs. The main duty of physicians caring for oncological patients is to optimize the analgesic treatment by achieving positive effects of the therapy and in the meantime minimizing the risk of side effects and addiction (abuse, drug addiction) $[25,30]$.

Non-pharmacological methods of pain relief are also noteworthy. However, they aim to support pharmacological treatment, not to replace it. Among these methods, we can distinguish support and the help of friends and family members, meetings with a psychologist or a priest, attending a supporting group for people with cancer, and getting to know people who effectively deal with pain during disease (graph 2) [1]. Although pain is the most common symptom of all cancers, it never occurs alone. Therefore, to improve the quality of life and mental health of patients, other symptoms should also be reduced [2, 3].

The importance of palliative care in the assessment and treatment of pain in patients with malignancy is worth mentioning. Palliative care is based on the cooperation of an interdisciplinary team, being responsible for the comprehensive management of the patient. Team members are focused on a patient's physical, psychological, social, and spiritual needs. Palliative care aims to maintain the highest possible comfort of life and control of the pain, which results in the reduction of the burden of the disease. Even though palliative medicine was historically considered as end-of-life care, recently it has been thought to be the caring for the patient from the moment of diagnosis for as long as needed [5].

\section{BREAST CANCER}

Pain and its psychological aspect among patients with breast cancer have been discussed in many studies and various methods have been used to evaluate it. The Brief Pain Inventory (BPI) is a self-completed questionnaire for pain assessment. The BPI scale is a numerical rating scale ranging from 0 to 10 and is often used in medical practice. It gives us two results: pain severity assessment and pain interference one [33].

The BPI scale was used to analyze the intensity of pain among women with metastatic breast cancer in the study conducted by Castel et al. The study showed that younger patients, 
non-Caucasians with an initial high score in Eastern Cooperative Oncology Group (ECOG) scale, with pre-existing skeletal-related events (SRE), and those without full-time employment are more exposed to the occurrence of severe pain [34].

A different study focused on examining the effect of the stress before surgical intervention in the treatment of breast cancer on the severity of side effects after the surgery. It was observed that the greater intensity of stress in patients significantly contributed to the intensification of postoperative pain, fatigue, and nausea [35].

Another study conducted in 2019 based on the BPI scale suggested that there is no correlation between the severity of the pain and the mental state of patients diagnosed with breast cancer. However, the authors of the study mentioned that a study group consisting of 36 patients is too small to draw an unambiguous conclusion [36].

Sipila et al. (2020) examined psychological subgroups of breast cancer patients. Among the various methods used to assess them, there was also the BPI scale. It revealed that patients prone to depressive states, cope with the pain less effectively. Psychological flexibility and optimism can significantly contribute to the successful management of pain in patients with breast cancer. Moreover, the use of artificial intelligence to identify such subgroups of patients may significantly affect the quality of the pain treatment by an interdisciplinary team [37].

\section{OVARIAN CANCER}

Ovarian cancer is the second most common gynecological cancer. In $75 \%$ of patients, it is diagnosed in the advanced clinical stage III or IV [38]. Its treatment regimen is based on surgery and chemotherapy. Both the disease and the treatment affect the occurrence and severity of symptoms and the quality of patients' lives [39]. Pain is one of the most common and bothersome symptoms - it is reported depending on the source among $60 \%$ [40] to about $87 \%$ of cases [41]. There is no standardized system for assessing pain and its impact on the quality of life of patients with ovarian cancer. Various scales, questionnaires, and their modifications are used in numerous studies that make it challenging to compare them. A promising indicator for the assessment of symptoms among patients with advanced ovarian cancer seems to be the National Comprehensive Cancer Network-Functional Assessment of Cancer Therapy - Ovarian Symptom Index (NFOSI-18), based on three scales - concerning symptoms related to the disease, treatment, and general well-being $[42,43]$.
In the 2017 study, Gilbertson-White et al. conducted a cross-sectional analysis of coping with pain, anxiety, and other aspects of the disease among 162 patients with ovarian cancer. The assessment used the Illness Perception Questionnaire (IPS), Symptom Representation Questionnaire (SRQ), Numeric Rating Scale (NRS), and Daily Copying Inventory (DCI). The mean pain intensity was rated by the patients at 5.5 on the NRS scale (0-10). Most of the respondents (96.9\%) reported discomfort related to pain. More than half $(63 \%)$ connected pain with disease or treatment. Moreover, the study shows that despite the use of an average of five pain coping strategies by the patients, they did not achieve satisfactory pain relief. Moreover, the experienced pain and the level of coping with it do not depend on the clinical situation of the patients, and are comparable among women with active disease, during treatment, or in remission [44].

Hwang et al., in turn, used a questionnaire developed based on Memorial Symptom Assessment Scale-Short Form (MSAS-SF), Hospital Anxiety and Depression Scale (HADS), Functional Assessment of Cancer Therapy-Ovarian (FACT-O), Functional Assessment of Chronic Illness Therapy (FACIT) to characterize symptoms, emotional state, quality of life and factors influencing pain among ovarian cancer patients undergoing chemotherapy. $87 \%$ of 192 patients reported pain, and fatigue, dissatisfaction as the most frequent symptoms. Study participants diagnosed 3 to 5 years earlier presented the highest levels of fatigue and pain. The study showed that the symptoms presented by the patients negatively affect various aspects of life. The anxiety and depression index among the respondents were high [41] and significantly exceeded the same indexes used in the study conducted among patients diagnosed with breast cancer [45].

\section{LUNG CANCER}

One of the malignancies with the highest incidence and mortality is lung cancer [46]. Patients with lung cancer experience a wide range of symptoms related to both the neoplastic process and treatment, especially in the advanced stage of the disease [47]. Pain in patients with lung cancer is both receptor-based and neuropathic and is also associated with frequent bone metastases [48]. For this reason, it has been the subject of many studies among these patients. Unfortunately, the assessment of its frequency, intensity and background is problematic and the results are various [49].

Potter and Higginson reviewed 32 studies on the prevalence, epidemiology and pathophysiology of pain in patients with lung cancer. 29 studies assessed the prevalence of pain, 
17 - its intensity, and 2 - its impact on everyday life and the level of discomfort. These studies used scales and questionnaires, such as: BPI, McGill Pain Questionnaire (MPQ), Memorial Pain Assessment Card (MPAC), Rotterdam Symptom Checklist (RSCL), Symptom Distress Scale (SDS). A review of studies showed that pain is the most common symptom among lung cancer patients and has a detrimental effect on psychosocial functioning. The cytological subtype of the neoplasm and gender did not seem to affect the experienced pain, but it occurred less frequently in older patients. The authors of the study suggest that it may be related to a lower pain perception or a lower tendency of older patients to complain about symptoms [50].

Morrison et al. (2017) focused on assessing emotional issues, quality of life, and symptom severity among 2205 lung cancer patients. The study found that younger patients, female smokers, unemployed people, advanced lung cancer patients, who were undergoing surgery or chemotherapy had greater emotional problems. Moreover, a strong correlation has been shown between emotional problems, low quality of life and the severity of symptoms, including pain [51]. Research also suggests that there is a correlation between the severity of symptoms and the level of hope in patients diagnosed with lung cancer. Patients characterized by a more optimistic way of thinking experienced less pain and other symptoms, as well as mental discomfort, anxiety disorders and depression $[52,53]$.

\section{COLON CANCER}

Colorectal cancer is the second most frequently diagnosed cancer among women and the third among men [54]. The basic form of treatment of non-disseminated colorectal cancer is surgery, supplemented with chemotherapy depending on the stage of the disease. Surgical treatment often results in pain and discomfort in the patient [55].

McCombie et al., using scales such as the Symptoms Distress Scale, Quality of Life Index, and the Global Quality of Life Scale, showed that patients receiving laparoscopic treatment compared to patients undergoing open colectomy are characterized by a higher quality of life and a lower intensity of postoperative pain [56].

In turn, Gonzalez-Saenz de Tejada et al. focused on the influence of psychosocial factors on the quality of life among 947 patients diagnosed with colorectal cancer one year after the surgery. Patients receiving social support, regardless of age, gender or profession, were characterized by higher independ- ence in everyday functioning and achieved higher scores in the quality-of-life questionnaires, which could have contributed to the lower incidence of depression and anxiety disorders in the respondents [57].

The key chemotherapeutic agent used in the treatment of colorectal cancer is oxaliplatin. A side effect of its use is acute peripheral polyneuropathy, which promotes the occurrence of depression and sleep disorders in patients, significantly reducing their quality of life [58]. Moreover, the intravenous infusion of chemotherapeutic agents, especially oxaliplatin, is associated with the occurrence of vascular pain, which is sometimes the reason why patients discontinue further treatment [59]. Another aspect that reduces the quality of life and increases the risk of pain is the need for stoma in patients who have undergone colon resection [60].

The study conducted at the Maria Sklodowska-Curie National Research Institute of Oncology among 238 patients diagnosed with colorectal cancer concerned the assessment, acceptance, adaptation to the disease and the strategy of coping with it. The following questionnaires were used in the study: Beliefs about Pain Control Questionnaire (BPCQ), Pain Coping Strategies Questionnaire (CSQ), Acceptance of Illness Scale (AIS), Mental Adjustment to Cancer Scale (Mini-MAC). Based on the BPCQ, which consists of three subscales measuring the strength of individual beliefs about pain treatment in the context of internal factors, the effectiveness of doctors, and random events, study participants assigned a key role in pain management to the internal factors - their own pain coping strategies and the level of acceptance of the disease. The level of acceptance of the disease in the respondents depended on the level of income per household member. The strategies of coping with the disease differed depending on the place of residence, professional status, or net income per family member. The increase in the subscales of internal factors was parallel to the increase in the level of education of patients [61].

\section{PANCREATIC CANCER}

Pancreatic cancer is associated with poor prognosis and its incidence is constantly increasing. It is a diagnostic challenge; therefore, it is often diagnosed at an advanced stage. Pain in the epigastric, mid-abdomen, back, or shoulder blade is one of the most common symptoms in addition to significant weight loss and jaundice [62]. It occurs among as many as $80 \%$ of those affected by this cancer, and $44 \%$ of them describe the pain as severe. Pain is a negative prognostic factor, and due to its severity and 
multidimensional nature, it is also a therapeutic challenge [63] Anxiety disorders and depression are more common in pancreatic cancer patients compared to the other neoplasms, and the severity of depression correlates with the severity of perceived pain [64]. Moreover, these disorders not only occur in patients experiencing disease and treatment but also often precede the onset of pancreatic cancer [65]. There are many theories explaining this phenomenon, however, there is no agreement as to the underlying mechanism. The causes were initially seen in the paraneoplastic syndrome, which produces a false neurotransmitter [66] Subsequently, mood disorders have been associated with disturbances in the secretory function of the pancreas [67], weakened immune system [68], and high levels of interleukin 6 [69]. There are many studies comparing various aspects of the disease and treatment in terms of the severity of symptoms and the patients standard of living. Hagiwara et al. in their work analyzed a group of patients receiving S-1 (oral fluoropyrimidine) and a group treated with gemcitabine. The first group showed a higher quality of everyday life, fewer problems with movement, as well as less pain and discomfort [70]. Other researchers have shown that the use of maintenance olaparib treatment does not reduce the quality of life in patients with BRCA mutation and disseminated pancreatic cancer [71]. Much space is also devoted to the systematization of pain treatment, as well as the search for new methods, as those currently used in many cases do not provide an acceptable alleviation of symptoms. In pain therapy, drugs from each step of the analgesic ladder, chemotherapy, as well as neurolysis, neuromodulation, radiotherapy, and ultrasounds are commonly used [63]. The studies indicate the high effectiveness of the visceral plexus blockade, which was manifested by a lower pain assessment on the VAS scale and a reduction in opiate consumption, with the simultaneous high safety of the procedure [72]. Chen et al. demonstrated a beneficial effect of the alternative method - electroacupuncture, on pain reduction in patients with pancreatic cancer [73].

\section{GASTRIC CANCER}

Gastric cancer is the fourth most common cancer in men and the fifth most common cancer in women. It is characterized by high mortality, reaching as much as $70 \%$ of cases [74]. Patients with advanced gastric cancer deal with general cachexia and local symptoms such as bleeding, obstruction, and pain. The methods for alleviating the latter include, in addition to pharmacological treatment, palliative radiotherapy, palliative chemotherapy, gastrectomy, bypass surgery, or endoscopic stenting [75]. Despite the unfavorable prognosis and a wide range of complaints accompanying patients diagnosed with gastric cancer, researchers from the Medical University of Hanoi found that the average quality of life of patients is higher than that of patients diagnosed with lung, breast, or colon cancer. Factors such as age, profession, education, and treatment methods had an impact on the patients' quality of life. Sexual activity was the sphere of life of the respondents whose quality was most decreased because of the disease [76].

Jentschura et al. focused their work on comparing the quality of life of patients after total and partial gastrectomy. Higher quality of life, probably dictated by lower weight loss and better general physical condition, characterized patients after subtotal surgery [77].

There have been many studies analyzing the influence of therapeutic and psychosocial factors on the quality of life and the severity of symptoms in patients with gastroenterological cancers. Due to the selectivity and lack of uniformity in the assessment of the quality-of-life level and the intensity of symptoms, their interpretation is ambiguous. It is a field for further research and analysis.

\section{CONCLUSIONS}

Pain is an important aspect of cancer that cannot be taken lightly. It affects both the lifestyle and quality of life, as well as the psyche of the patient and the people around him. Pain is the most common symptom of cancer and is largely responsible for the problem of coping with the disease.

Appropriate scales and questionnaires such as BPI, MPQ, MPAC, RSCL, SDS, NRS, or ECOG are very useful for the proper assessment of pain and the degree of efficiency during disease. They allow not only the assessment of pain but also to introduce the appropriate treatment to fight ailments. Effective pain relief is extremely important, as appropriate analgesia allows for an easier bearing of the effects of the disease and improves the mental condition of patients and their relatives. 


\section{References}

1. Van den Beuken-van Everdingen M, Hochstenbach $L$, Joosten E et al. Update on Prevalence of Pain in Patients With Cancer: Systematic Review and Meta-Analysis. J Pain Symptom Manage. 2016; 51(6): 1070-90.

2. Porter L, Keefe F. Psychosocial issues in cancer pain. Curr Pain Headache Rep. 2011; 15(4): 263-70.

3. Breivik H, Cherny N, Collett B et al. Cancer-related pain: a pan-European survey of prevalence, treatment, and patient attitudes. Ann Oncol. 2009; 20(8): 1420-33.

4. Yang J, Bauer BA, Wahner-Roedler DL et al. The Modified WHO Analgesic Ladder: Is it Appropriate for Chronic Non-Cancer Pain? J Pain Res. 2020; 13: 411-7.

5. Portenoy R. Treatment of cancer pain. Lancet. 2011; 377(9784): 2236-47.

6. Leppert W, Zajaczkowska R, Wordliczek J et al. Pathophysiology and clinical characteristics of pain in most common locations in cancer patients. J Physiol Pharmacol. 2016; 67(6): 787-99.

7. Russo MM, Sundaramurthi T. An Overview of Cancer Pain: Epidemiology and Pathophysiology. Semin Oncol Nurs. 2019; 35(3): 223-8

8. Yoon SY, Oh J. Neuropathic cancer pain: prevalence, pathophysiology, and management. Korean J Intern Med. 2018; 33(6): 1058-69.

9. Jensen TS, Finnerup NB. Allodynia and hyperalgesia in neuropathic pain: clinical manifestations and mechanisms. Lancet Neurol. 2014; 13(9): 924$-35$.

10. Tsuda M. New approach for investigating neuropathic allodynia by optogenetics. Pain. $2019 ; 160$ (suppl 1):53-8.

11. Davis MP. Cancer-Related Neuropathic Pain: Review and Selective Topics. Hematol Oncol Clin North Am. $2018 ; 32(3): 417-31$.

12. Jongen JL, Hujisman ML, Jessurun J et al. The evidence for pharmacologic treatment of neuropathic cancer pain: beneficial and adverse effects. J Pain Symptom Manage. 2013; 46(4): 581-90.e1.

13. Sisignano M, Baron R, Scholich K et al. Mechanism-based treatment for chemotherapy-induced peripheral neuropathic pain. Nat Rev Neurol. 2014 10(12): 694-707.

14. Müller-Schwefe $G$, Ahlbeck K, Aldington D et al. Pain in the cancer patient: different pain characteristics CHANGE pharmacological treatment requirements. Curr Med Res Opin. 2014; 30(9): 1895-908.

15. Finnerup NB, Attal N, Haroutounian S et al. Pharmacotherapy for neuropathic pain in adults: a systematic review and meta-analysis. Lancet Neurol. 2015; 14(2): 162-73.

16. Smith $\mathrm{EM}$, Pang $\mathrm{H}$, Cirrincione $\mathrm{C}$ et al. Effect of duloxetine on pain, function, and quality of life among patients with chemotherapy-induced painful peripheral neuropathy: a randomized clinical trial. JAMA. 2013; 309(13): 1359-67.

17. Kim HK, Kwon JY, Yoo C et al. The Analgesic Effect of Rolipram, a Phosphodiesterase 4 Inhibitor, on Chemotherapy-Induced Neuropathic Pain in Rats. Anesth Analg. 2015; 121(3): 822-8.

18. Kuyrukluyıldız U, Kupeli I, Bedir Z et al. The Effect of Anakinra on Paclitaxel-Induced Peripheral Neuropathic Pain in Rats. Turk J Anaesthesiol Reanim. 2016; 44(6): 287-94.

19. Araki M, Matsuoka T, Miyamoto K et al. Efficacy of the anti-IL-6 receptor antibody tocilizumab in neuromyelitis optica: a pilot study. Neurology. 2014; 82(15): 1302-6.

20. Prescott S A, Ratté S. Chapter 23 - Somatosensation and Pain, in Conn's Translational Neuroscience. Conn PM (ed). San Diego, Academic Press 2017; 517-39.

21. Nijs J, Leysen L, Adriaenssens $\mathrm{N}$ et al. Pain following cancer treatment: Guidelines for the clinical classification of predominant neuropathic, nociceptive and central sensitization pain. Acta Oncol. 2016; 55(6): 659-63.

22. Schmidt BL, Hamamoto DT, Simone DA et al. Mechanism of cancer pain. Mol Interv. 2010; 10(3): 164-78.

23. Bechakra M, Moerdijk F, van Rosmalen J et al. Opioid responsiveness of nociceptive versus mixed pain in clinical cancer patients. Eur J Cancer. 2018, 105: 79-87.

24. Van den Beuken-van Everdingen $M$, de Rijke J, Kessels A et al. Prevalence of pain in patients with cancer: a systematic review of the past 40 years. Ann Oncol. 2007; 18(9): 1437-49.

25. Greco M, Roberto A, Corli O et al. Quality of cancer pain management: an update of a systematic review of undertreatment of patients with cancer. J Clin Oncol. 2014; 32(36): 4149-54.

26. Hastie B, Gilson A, Maurer M et al. An examination of global and regional opioid consumption trends 1980-2011. J Pain Palliat Care Pharmacother. 2014; 28(3): 259-75.

27. Deandrea S, Corli O, Consonni D et al. Prevalence of Breakthrough Cancer Pain: A Systematic Review and a Pooled Analysis of Published Literature. J Pain Symptom Manage. 2014; 47(1): 57-76.

28. Magee D, Bachtold S, Brown M et al. Cancer pain: where are we now? Pain Manag. 2019; 9(1): 63-79.

29. Syrjala K, Jensen M, Mendoza M et al. Psychological and behavioral approaches to cancer pain management. J Clin Oncol. 2014; 32(16): 1703-11.

30. Imam M, Kuo A, Ghassabian S et al. Progress in understanding mechanisms of opioid-induced gastrointestinal adverse effects and respiratory depression. Neuropharmacology. 2018; 15(131): 238-55.

31. Pannunzio A, Coluccia M. Cyclooxygenase-1 (COX-1) and COX-1 Inhibitors in Cancer: A Review of Oncology and Medicinal Chemistry Literature. Pharmaceuticals (Basel). 2018; 11(4): 101.

32. Magee DJ, Jhanji S, Poulogiannis G et al. Nonsteroidal anti-inflammatory drugs and pain in cancer patients: a systematic review and reappraisal of the evidence. Br J Anaesth. 2019; 123(2): e412-e23.

33. Poquet N, Lin C. The Brief Pain Inventory (BPI). J Physiother. 2016; 62(1): 52.

34. Castel L, Abernethy A, Li Y et. al. Hazards for Pain Severity and Pain Interference with Daily Living, with Exploration of Brief Pain Inventory Cutpoints, Among Women with Metastatic Breast Cancer. J Pain Symptom Manage. 2007; 34(4): 380-92.

35. Montgomery G, Schnur J, Erblich J et al. Pre-surgery Psychological Factors Predict Pain, Nausea, and Fatigue One Week After Breast Cancer Surgery. J Pain Symptom Manage. 2010; 39(6): 1043-52.

36. Cindy C, Wijovi F, Putri H et al. Correlation between Cancer Pain and Psychological Health Problems in Breast Cancer Patients: A Preliminary Study. Ann Oncol. 2019; 30(6): 146.

37. Sipila R, Kalso E, Lotsch J. Machine-learned identification of psychological subgroups with relation to pain interference in patients after breast cancer treatments. Breast. 2020; 50: 71-80.

38. NCI SEER Database. SEER Stat Fact Sheets: Ovary Cancer. 2015. http://seer.cancer.gov/statfacts/html/ovary.html (access: 20.09.2020).

39. American Cancer S. Cancer Facts \& Figures 2015. American Cancer Society. 2015. https://www.cancer.org/research/cancer-facts-statistics/all-cancer-facts-figures/cancerfactsfigures-2015.html (access: 20.09.2020).

40. Stavraka C, Ford A, Ghaem-Maghami S et al. A study of symptoms described by ovarian cancer survivors. Gynecol Oncol. 2012; $125(1)$ : 59-64.

41. Kyung-Hye H, Ok-Hee C, Yang-Sook Y. Symptom clusters of ovarian cancer patients undergoing chemotherapy, and their emotional status and quality of life. Eur J Oncol Nurs. 2016; 21:215-22.

42. Katumba J, Obore S, Kaye DK. Health-related quality of life among patients with ovarian cancer at Mulago Hospital, Uganda. Int J Gynecol Obstet. 2013; 122(2): 115-7.

43. Shaunfield S, Jensen S, Fisher A et al. Further content validation of the 18-itemNCCN/FACT Ovarian Symptom Index and its Disease Related Symptom-Physical(DRS-P) subscale for use in advanced ovarian cancer clinical trials. Health Qual Life Outcomes. 2019; 17(1): 185. 
44. Gilbertson-White S, Campbell G, Ward S et al. Coping with Pain Severity, Distress, and Consequences in Women with Ovarian Cancer. Cancer Nurs. 2017; 40(2): 117-23.

45. So W, Marsh G, Ling W et al. The symptom cluster of fatigue, pain, anxiety, and depression and the effect on the quality of life of women receiving treatment for breast cancer: a multicenter study. Oncol Nurs Forum. 2009; 36(4): E205-14.

46. Mao Y, Yang D, He J et al. Epidemiology of Lung Cancer. Surg Oncol Clin N Am. 2016; 25(3): 439-45.

47. Barbera L, Paszat L, Qiu F. End-of-life care in lung cancer patients in Ontario: Aggressiveness of care in the population and a description of hospital admissions. J Pain Symptom Manage. 2008; 35(3): 267-74.

48. Zajączkowska R, Kocot-Kępska M, Leppert W et al. Bone Pain in Cancer Patients: Mechanisms and Current Treatment. Int J Mol Sci. 2019; 20(23): 6047.

49. Polański J, Jankowska-Polańska B, Mazur G et al. Strategies of Coping with Pain: Differences Associated with the Histological Type of Lung Cancer. Adv Exp Med Biol. 2019; 1222: 43-53. https://doi.org/10.1007/5584_2019_429.

50. Potter J, Higginson J. Pain experienced by lung cancer patients: a review of prevalence, causes and pathophysiology. Lung Cancer. 2004 ; $43: 247-57$. https://doi.org/10.1016/j.lungcan.2003.08.030.

51. Morrison E, Novotny P, Sloan J et al. Emotional Problems, Quality of Life, and Symptom Burden in Lung Cancer Patients. Clin Lung Cancer. 2017; 18(5): 497-503. https://doi.org/10.1016/j.cllc.2017.02.008.

52. Berendes D, Keefe F, Somers T. Hope in the context of lung cancer: relationships of hope to symptoms and psychological distress. J Pain Symptom Manage. 2010; 40(2): 174-82. https://dx.doi.org/10.1016\%2Fj.jpainsymman.2010.01.014.

53. Steffen L, Cheavens J, Vowles $\mathrm{K}$ et al. Hope-related goal cognitions and daily experiences of fatigue, pain, and functional concern among lung cancer patients. Support Care Cancer. 2020; 28(2): 827-35.

54. Bray F, Ferlay J, Soerjomataram I et al. Global cancer statistics 2018: GLOBOCAN estimates of incidence and mortality worldwide for 36 cancers in 185 countries. CA Cancer J Clin. 2018; 68: 394-424.

55. Bertelsen CA, Larsen HM, Neuenschwander AU et al. Long-term Functional Outcome After Right-Sided Complete Mesocolic Excision Compared With Conventional Colon Cancer Surgery: A Population-Based Questionnaire Study. Dis Colon Rectum. 2018; 61(9): $1063-72$.

56. McCombie A, Frizelle F, Bagshaw F et al. The ALCCaS Trial: A Randomized Controlled Trial Comparing Quality of Life Following Laparoscopic Versus Open Colectomy for Colon Cancer. Dis Colon Rectum. 2018; 61(10): 1156-62.

57. Gonzalez-Saenz de Tejada M, Bilbao A, Baréb M et al. Association between social support, functional status and change in Health Related Quality of Life and changes in anxiety and depression in colorectal cancer patients. Psychooncology. 2017; 26(9): 1263-9.

58. Mols F, Beijers T, Lemmens V et al. Chemotherapy-induced neuropathy and its association with quality of life among 2- to 11-year colorectal cancer survivors: results from the population-based PROFILES registry. J Clin Oncol. 2013; 31(21): 2699-707.

59. Matsuoka T, Yoshida Y, Aisu N et al. Evaluation of vascular pain in patients with colorectal cancer receiving peripheral venous chemotherapy with or without oxaliplatin. Sci Rep. 2019; 9(1): 1819.

60. Vonk-Klaassen $\mathrm{S}$, de Vocht $\mathrm{H}$, den Ouden $\mathrm{M}$ et al. Ostomy-related problems and their impact on quality of life of colorectal cancer ostomates: a systematic review. Qual Life Res. 2016; 25(1): 125-33.

61. Czerw A, Religioni U, Deptała A et al. Assessment of pain, acceptance of illness, adjustment to life with cancer, and coping strategies in colorectal cancer patients. Prz Gastroenterol. 2016; 11(2): 96-103.

62. McGuigan A, Kelly P, Turkington R et al. Pancreatic cancer: A review of clinical diagnosis, epidemiology, treatment and outcomes. World J Gastroenterol. 2018; 24(43): 4846-61.

63. Koulouris A, Banim P, Hart A. Pain in Patients with Pancreatic Cancer: Prevalence, Mechanisms, Management and Future Developments. Dig Dis Sci. 2017: 62(4): 861-70

64. Kelsen D, Portenoy R, Thaler H et al. Pain and depression in patients with newly diagnosed pancreas cancer. J Clin Oncol. 1995; 13(3): 748-55

65. Kenner B. Early Detection of Pancreatic Cancer: The Role of Depression and Anxiety as a Precursor for Disease. Pancreas. $2018 ; 47(4)$ : 363-7.

66. Parker G, Brotchie H. Pancreatic Cancer and Depression: A Narrative Review. J Nerv Ment Dis. 2017; 205(6): 487-90.

67. Barnes AF, Yeo TP, Leiby B et al. Pancreatic Cancer-Associated Depression: A Case Report and Review of the Literature. Pancreas. $2018 ; 47(9)$ : 1065-77.

68. Arehart-Treichel J. Several factors may explain cancer, Depression Link. Published online 2003. http://psychnews.psychiatryonline.org (access: 25.09.2020)

69. Breitbart W, Rosenfeld B, Tobias K et al. Depression, cytokines, and pancreatic cancer. Psychooncology. 2014; 23(3): 339-45.

70. Hagiwara Y, Ohashi Y, Uesaka K et al. Health-related quality of life of adjuvant chemotherapy with S- 1 versus gemcitabine for resected pancreatic cancer: Results from a randomised phase III trial (JASPAC 01). Eur J Cancer. 2018; 93: 79-88.

71. Hammel $\mathrm{P}$, Kindler $\mathrm{H}$, Reni $\mathrm{M}$ et al. Health-related quality of life in patients with a germline BRCA mutation and metastatic pancreatic cancer receiving maintenance olaparib. Ann Oncol. 2019; 30(12): 1959-68.

72. Molnár I, Hegyi G, Zsom L. Celiac plexus block increases quality of life in patients with pancreatic cancer. J Pain Res. 2019; 12: $307-15$.

73. Chen H, Liu TY, Kuai L et al. Electroacupuncture treatment for pancreatic cancer pain: a randomized controlled trial. Pancreatology. 2013; 13(6): 594-97.

74. Guggenheim D, Shah M. Gastric Cancer Epidemiology and Risk Factors. J Surg Oncol. 2013; 107: 230-6.

75. Tey J, Yang Soon Y, Yao Koh W et al. Palliative radiotherapy for gastric cancer: a systematic review and meta-analysis. Oncotarget. 2017; 8(15): 25797-805.

76. Thi Dang D, Thi Nguyen L, Thi Dang N et al. Quality of Life in Vietnamese Gastric Cancer Patients. Biomed Res Int. 2019.

77. Jentschura D, Winkler M, Strohmeier N et al. Quality-of-life after curative surgery for gastric cancer: a comparison between total gastrectomy and subtotal gastric resection. Hepatogastroenterology. 1997; 44(16): 1137-42.

Authors' contributions:

Izabela Marzec: 40\%; Kinga Grabska: 40\%; Marta M. Fudalej: 10\%; Anna M. Badowska-Kozakiewicz: 10\%.

Conflict of interests:

None.

Financial support:

There was no financial support

Ethics:

The authors had full access to the data and take full responsibility for its integrity.

All authors have read and agreed with the content of the manuscript as written.

The paper complies with the Helsinki Declaration, EU Directives and harmonized requirements for biomedical journals. 